> Les femmes vivent désormais plus d'un tiers de leur vie après la survenue de la ménopause. Le déclin de la production d'œstrogènes endogènes au cours de cette période s'accompagne fréquemment de troubles fonctionnels qui affectent la qualité de vie. Ces symptômes peuvent être soulagés par un traitement hormonal (THM) initialement fondé sur l'administration d'œstrogènes conjugués équins (principalement aux États-Unis, par voie orale) ou d'un œstrogène naturel, le 17 $\beta$-estradiol (en Europe, notamment par voie transdermique). Le récepteur des œstrogènes $\alpha(R \varepsilon \alpha)$ relaye la majorité des effets physiologiques des œstrogènes. RE $\alpha$ appartient à la superfamille des récepteurs nucléaires. II régule la transcription de gènes via ses fonctions activatrices (AFI et AF2). Outre ces actions génomiques classiques, les œstrogènes peuvent aussi activer une sous-population de récepteurs $R \varepsilon \alpha$ présents à la membrane des cellules et ainsi induire des signaux rapides. Dans cette revue, nous résumerons l'évolution des THM depuis les débuts de la substitution hormonale jusqu'aux nouvelles molécules émergentes fondées sur une modulation sélective du REQ. Nous décrirons également les progrès récents sur la compréhension des mécanismes d'action des œstrogènes, en détaillant les rôles respectifs des REa nucléaire et membranaire et les développements thérapeutiques possibles qui pourraient en découler. <

Les œstrogènes sont à l'origine de nombreuses réponses physiologiques. Ces hormones jouent un rôle essentiel dans les modifications qui apparaissent lors de la puberté, en particulier la croissance du vagin, de l'utérus, des trompes de Fallope et de la glande mammaire. Au cours du cycle menstruel, elles sont finement

Vignette (๔ Canopé / CNDP / Universcience / MGEN / Inserm / Educagri).

\section{Vers une \\ optimisation \\ de la modulation \\ du récepteur \\ des cstrogènes \\ dans le traitement \\ hormonal de \\ la ménopause}

Marie-Cécile Valéra, Coralie Fontaine,

Emmanuelle Noirrit-Esclassan, Frédéric Boudou,

Melissa Buscato, Marine Adlanmerini,

Florence Trémollières, Pierre Gourdy,

Françoise Lenfant, Jean-François Arnal

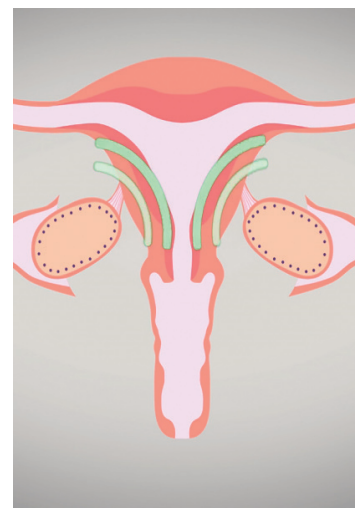

Inserm U1048 et université

Toulouse III, I2MC, CHU Rangueil, BP 84225, 31432 Toulouse Cedex 4 , France.

francoise.lenfant@inserm.fr

régulées afin de rendre l'environnement favorable à la fécondation et à l'implantation de l'embryon. Au cours de la grossesse, leurs niveaux augmentent. Elles stimulent la maturation fotale et favorisent le maintien de la grossesse. Elles ont également des effets importants sur les voies urinaires, le système cardiovasculaire, le tissu osseux, la peau, les muqueuses, le cerveau où elles modulent de nombreux comportements (sexuel et alimentaire notamment) [1]. Les ovaires sont la principale source d'œstrogènes circulants chez les femmes avant la ménopause, le 17- $\beta$ œstradiol ( $\varepsilon 2$ ) étant la principale hormone endogène. Dans le sérum, ils se lient à des protéines (SHBG [sex hormone-binding globulin] et albumine) et leurs effets biologiques dépendent de leur liaison à deux récepteurs des œstrogènes $(R \varepsilon): R \varepsilon \alpha$ et $R \varepsilon \beta$.

Dans les pays industrialisés, l'âge médian des femmes à la ménopause se situe entre 50 et 52 ans. La ménopause est définie comme la fin permanente de la menstruation et de l'ovulation, due à l'insuffisance ovarienne. Elle est donc caractérisée par l'arrêt de la production d'œstrogènes par les ovaires. Les tissus adipeux, cérébraux et osseux ainsi que les vaisseaux peuvent cependant produire de petites quantités d'œstrogènes, par aromatisation de la testostérone en $\varepsilon 2$, qui agissent le plus souvent localement mais aussi, parfois, par voie systémique. À la ménopause, 
la baisse des niveaux d'œstrogènes est souvent à l'origine d'un certain nombre de symptômes, dont les plus courants sont les bouffées de chaleur, les sueurs nocturnes et les troubles du sommeil, les douleurs ostéoarticulaires [1, 2]. Ils sont souvent associés à des troubles de l'humeur, de la mémoire, de la concentration. La ménopause favorise également l'ostéoporose et donc une augmentation du risque de fracture osseuse, mais aussi d'événements coronariens et de troubles métaboliques. Elle peut induire un syndrome génito-urinaire, qui correspond à une atrophie vulvovaginale et à des symptômes du bas appareil urinaire [3]. Ces troubles sont habituellement soulagés par l'administration d'un traitement hormonal de la ménopause (THM) [2]. Nous donnerons dans cette revue un aperçu historique de l'évolution des différents THM au cours des dernières décennies. Nous décrirons également comment les avancées obtenues pour la compréhension de la modulation du REa permettent maintenant de concevoir de nouveaux traitements.

\section{Des débuts du traitement hormonal de la ménopause jusqu'à la grande étude WHI (Women's Health Initiative)}

Les œstrogènes sont utilisés depuis les années 1940, et leur prescription a augmenté au cours des années 1960. Les premières inquiétudes quant à ces traitements sont apparues dans les années 1970, lorsque le lien entre la prescription d'œstrogènes seuls comme THM et l'augmentation du risque de cancer de l'endomètre a été révélé. Les œstrogènes oraux sont depuis associés à un progestatif, pour prévenir l'hyperplasie de l'endomètre à l'origine du sur-risque de cancer [4]. Le THM, d'abord prescrit pour limiter les symptômes de la ménopause, a ensuite eu comme objectif de ralentir le vieillissement de certains tissus. Plusieurs études avaient montré que ce traitement était associé à la prévention de maladies coronariennes, de l'ostéoporose et de la maladie d'Alzheimer [5]. En raison de la vaste utilisation du THM dans les années 1990, les instituts américains de la santé (le NIH, National institutes of health) ont alors lancé une large étude randomisée - la WHI (Women's Health Initiative) - afin d'évaluer précisément les avantages et les risques associés à de tels traitements. En 2002, les premiers résultats de cet essai clinique, qui incluait environ 16000 femmes recevant le traitement par des œstrogènes conjugués équins ( $\varepsilon \subset \varepsilon$, un mélange d'œstrogènes extraits de l'urine de juments gravides) en combinaison avec un progestatif de synthèse (l'acétate de médroxyprogestérone), ont été publiés après un suivi moyen de 5,2 ans. Les conclusions immédiates ont été que cette combinaison hormonale était associée à une incidence accrue de maladie thromboembolique veineuse (MTEV) et de cancers du sein et, de manière plus inattendue, à une augmentation des maladies coronariennes que ce traitement était pourtant censé prévenir [6]. Le deuxième volet de cette étude intéressait des femmes ménopausées hystérectomisées qui recevaient soit de l'ECE seul, soit un placebo. Cet essai n'a montré aucune aggravation du risque coronarien, et une légère diminution de l'apparition de cancer du sein après un suivi de près de 7 ans [7]. Pourtant, une tendance à l'augmentation du risque de MTEV et une augmentation significative du risque d'accident vasculaire cérébral ont été observées chez les femmes hystérectomisées traitées par દCE seul [7] (Tableau I).
À l'issue de cela, la prescription de THM a rapidement diminué, malgré les limites majeures de l'étude WHI, en particulier en raison de l'âge des participantes, qui étaient pour la plupart éloignées de l'âge de la ménopause. Près d'un quart des femmes de cette étude étaient âgées de plus de 70 ans. C'est pourtant dans ce sous-groupe de femmes que la combinaison d'œstrogènes et de progestatif favorisait les événements coronariens, alors que les femmes plus jeunes (de moins de 60 ans) présentaient une incidence coronarienne similaire au placebo [6]. Ces résultats, ainsi que la publication d'essais cliniques spécifiquement conçus pour tester l'impact du délai de prescription, suggèrent que le THM peut être utilisé avec un rapport risque-bénéfice favorable dans les 5 à 10 premières années suivant le début de la ménopause $[8,9]$. La voie d'administration des estrogènes tout comme la nature du progestatif associé se sont révélées être des déterminants importants du risque de maladie thromboembolique veineuse (MTEV) [10], voire du risque d'accident vasculaire cérébral [11]. II existe, en particulier, un contraste frappant entre les pratiques américaines et européennes en ce qui concerne la nature du progestatif utilisé. Aux États-Unis, I'utilisation de l'acétate de médroxyprogestérone a un impact défavorable sur le risque coronarien et mammaire. Au contraire, une grande variété de progestatifs, tels que la progestérone naturelle micronisée ou les dérivés de prégnane, sont prescrits dans les pays européens [12]. Par ailleurs, les études sur l'incidence de la voie d'administration montrent un risque accru de MTEV chez les utilisatrices du THM par voie orale, contrairement à la voie transdermique [12]. Cet effet semble attribuable au premier passage hépatique, à l'origine d'une augmentation des facteurs de coagulation du sang circulant qui n'est observée qu'avec la voie orale [13] (Tableau I). L'identification d'associations plus sûres est donc nécessaire afin d'améliorer la balance bénéfice/risque chez les patientes sous THM.

\section{Du SERM au TSEC : vers une modulation sélective du récepteur des œstrogènes pour améliorer la balance bénéfice-risque du THM}

Le traitement idéal devrait préserver les effets bénéfiques des œstrogènes sur le tissu osseux (croissance, maturation et régulation de son remodelage) et sur les systèmes uro-génital, métabolique et vasculaire et réduire les effets indésirables (notamment le risque de MTEV et celui de cancer du sein). Dans cette optique, des modulateurs sélectifs des récepteurs des œstrogènes (les SERM) sont développés depuis plus 


\begin{tabular}{|c|c|c|c|}
\hline & & Indication & Principaux effets secondaires \\
\hline \multirow{3}{*}{ CEstrogènes } & $\begin{array}{l}\text { Estradiol + progestatif/ } \\
\text { progestérone } \\
\text { Voies non orale et orale } \\
\text { (Europe) }\end{array}$ & $\begin{array}{l}\text { Symptômes du climatère chez les } \\
\text { femmes non hystérectomisées } \\
\text { Prévention de l'ostéoporose }\end{array}$ & $\begin{array}{l}\text { Cancer du sein } \\
\text { Pas d'augmentation du risque cardiovasculaire et } \\
\text { thrombotique (voie non orale seule) dans les études } \\
\text { de cohortes (doit être confirmée par des études } \\
\text { randomisées) }\end{array}$ \\
\hline & $\begin{array}{l}\text { Estrogènes conjugués équins } \\
\text { Voie orale (États-Unis) }\end{array}$ & $\begin{array}{l}\text { Symptômes du climatère chez } \\
\text { les femmes hystérectomisées } \\
\text { Prévention de l'ostéoporose }\end{array}$ & $\begin{array}{l}\text { Études randomisées: } \\
\text { Accident vasculaire cérébral } \\
\text { Risque de thrombose veineuse } \\
\text { Études randomisées: }\end{array}$ \\
\hline & $\begin{array}{l}\text { Estrogènes conjugués équins } \\
+ \text { progestatif } \\
\text { Voie orale (États-Unis) }\end{array}$ & $\begin{array}{l}\text { Symptômes du climatère chez les } \\
\text { femmes non hystérectomisées } \\
\text { Prévention de l'ostéoporose }\end{array}$ & $\begin{array}{l}\text { Accident vasculaire cérébral } \\
\text { Risque de thrombose veineuse } \\
\text { Cancer du sein } \\
\text { Accidents coronariens (>70 ans) }\end{array}$ \\
\hline \multirow[b]{2}{*}{ SERM } & Tamoxifène & Cancer du sein & $\begin{array}{l}\text { Hyperplasie endométriale } \\
\text { Risque de thrombose veineuse }\end{array}$ \\
\hline & $\begin{array}{l}\text { Raloxifène } \\
\text { Bazedoxifène }\end{array}$ & $\begin{array}{l}\text { Prévention et traitement de } \\
\text { l'ostéoporose chez la femme } \\
\text { ménopausée } \\
\text { Traitement de l'ostéoporose } \\
\text { chez la femme ménopausée }\end{array}$ & $\begin{array}{l}\text { Profil endométrial favorable } \\
\text { Risque de thrombose veineuse }\end{array}$ \\
\hline TSEC & $\begin{array}{c}\text { Bazédoxifène }+ \text { œstrogènes } \\
\text { conjugués équins }\end{array}$ & $\begin{array}{l}\text { Symptômes du climatère chez les } \\
\text { femmes non hystérectomisées } \\
\text { Prévention de l'ostéoporose }\end{array}$ & $\begin{array}{l}\text { Profil endométrial favorable } \\
\text { Risque de thrombose veineuse? } \\
\text { Cancer du sein? (Études in vivo favorables) }\end{array}$ \\
\hline
\end{tabular}

Tableau I. Indications et risques associés des principales molécules utilisées dans le traitement hormonal de la ménopause. SERM : selective estrogen receptor modulators ; TSEC : tissue-selective estrogen complex.

d'un demi-siècle, ouvrant de nouvelles perspectives d'intervention. Les SERM se lient aux RE avec une haute affinité. Ils se caractérisent par des actions spécifiques en mimant l'action des œestrogènes sur certains tissus (action agoniste) ou, au contraire, en bloquant leurs effets sur d'autres organes (action antagoniste), en particulier le tissu mammaire [14].

L'un des SERM les plus anciens est le tamoxifène. II a été initialement développé comme nouveau contraceptif mais il s'est finalement imposé dans le traitement du cancer du sein. II agit principalement comme un antagoniste des œstrogènes sur le tissu mammaire et prévient la récidive du cancer du sein. En revanche, il mime l'action des œstrogènes au niveau de l'os, mais aussi, dans une certaine mesure, sur l'utérus (avec, de fait, un certain risque d'hyperplasie endométriale et de cancer de l'endomètre). II augmente, comme les estrogènes oraux, le risque de MTEV [15]. Malgré son action favorable sur le risque athéromateux et dans la prévention de l'infarctus du myocarde [16], ses effets secondaires se traduisent par une induction de bouffées de chaleur et des modifications de l'endomètre associées à un faible sur-risque de cancer de l'utérus (Tableau I).

Le raloxifène est un SERM de deuxième génération prescrit dans la prévention et le traitement de l'ostéoporose chez les femmes méno- pausées aux États-Unis et en Europe. Ni le tamoxifène ni le raloxifène n'ont d'effets bénéfiques sur les symptômes climatériques, en particulier les bouffées de chaleur, et tous deux augmentent le risque de MTEV $[17,18]$. Le raloxifène n'induit pas, contrairement au tamoxifène, de stimulation de l'endomètre, et donc de sur-risque de cancer de l'endomètre, et son effet protecteur vis-à-vis du cancer du sein est moindre. Il est également moins à risque que le tamoxifène en ce qui concerne les atteintes ophtalmologiques (augmentation des cataractes) ou les lithiases vésiculaires [19]. Le bazédoxifène (BZA) est un SERM de troisième génération qui mime les effets favorables des œstrogènes sur l'os et le métabolisme lipidique (effets agonistes sur ces tissus) [20]. II a été approuvé aux États-Unis pour le traitement de l'ostéoporose postménopausique. Il a en outre des activités antagonistes sur le sein et l'utérus [21] : dans ces 2 tissus, il bloque l'action des œstrogènes en induisant la dégradation du REQ. Cela constitue un mécanisme original permettant d'empêcher l'action des œstrogènes sur ces 
deux cibles sexuelles [22]. Dans le foie, le BZA dégrade aussi le RE $\alpha$ après un traitement aigu, bloquant l'action de l'E2 ou des $દ C \varepsilon$ dans ce tissu. En revanche, après un traitement chronique, la dégradation du RE$\alpha$ par le BZA reste effective dans l'utérus mais pas dans le foie. Le BZA administré de façon chronique acquiert alors une activité agoniste, qu'il soit administré seul ou en combinaison avec l'ECE, se traduisant par l'induction de la transcription de gènes cibles du REa dans le foie [23]. Cette action hépatique du BZA pourrait contribuer à la protection métabolique que présente ce SERM dans des modèles murins d'obésité et de diabète de type 2 [20]. Ces études mettent en évidence l'originalité du BZA, dont l'action agoniste/antagoniste varie en fonction des tissus et du temps d'administration de la molécule. Néanmoins, dans une étude randomisée menée sur des femmes postménopausées, une incidence accrue de MTEV, très similaire à celle observée avec les autres SERM, a été observée dans les groupes traités par le BZA par rapport aux groupes non traités [24] (Tableau I).

Le concept de TSEC (tissue selective estrogen complex) consiste à associer un SERM à un ou plusieurs œstrogènes [25] afin d'optimiser les bénéfices de chacune des molécules prises séparément. Dans des études cliniques réalisées en double aveugle, l'association du BZA et de l'દCદ a entraîné une amélioration significative des symptômes climatériques : bouffées de chaleur, troubles du sommeil, atrophie vaginale avec une amélioration de la qualité de vie par rapport aux femmes recevant le placebo. Cette association permet également d'inhiber la résorption osseuse et de prévenir la perte osseuse post-ménopausique sans induire d'hyperplasie de l'endomètre et sans modification de la densité mammaire [26, 27]. Dans un essai randomisé comparant l'ECE associé au BZA ou à un placebo chez 2000 femmes, aucune différence entre les 2 groupes n'a été observée en ce qui concerne le risque de $M T \varepsilon V$, mais la puissance de cet essai n'était pas suffisante pour conclure [28]. Dans cette étude, les facteurs de coagulation se sont néanmoins avérés légèrement modifiés par le traitement, mais aucun effet marquant de l'association entre $\varepsilon C \varepsilon$ et BZA concernant les facteurs de coagulation d'origine hépatique n'a été observé [28]. Concernant le risque de cancer du sein, des études réalisées in vitro [29] et des expériences in vivo sur des modèles de cancer du sein [30] montrent un effet protecteur de l'association de l' $\varepsilon C \varepsilon$ et du BZA. Dans la mesure où les SERM diminuent le risque d'apparition du cancer du sein chez la femme, on pourrait raisonnablement envisager, sinon un effet bénéfique, au moins une neutralité d'effet lorsque associés à l'દCદ. Cela reste néanmoins à confirmer par une étude clinique randomisée (Tableau I).

La combinaison entre BZA et $\varepsilon C \varepsilon$ est actuellement le premier traitement oral de THM à base d'œstrogènes non associés à un progestatif approuvé par la FDA (Food and drug administration) aux États-Unis, pour soulager les symptômes vasomoteurs modérés à sévères et prévenir la déminéralisation osseuse chez les femmes ménopausées non hystérectomisées [31]. Ce traitement a été commercialisé aux ÉtatsUnis sous le nom de Duavee ${ }^{\circledR}$ et a également été approuvé par l'Agence européenne des médicaments (pour la seule indication des troubles climatériques en Europe). Cependant, sa mise sur le marché en France est à ce jour un échec, notamment en raison de l'utilisation des œstro- gènes conjugués équins par voie orale, la préférence allant à la voie transdermique en raison de son moindre risque thrombotique.

\section{Une nouvelle dimension : la modulation sélective du RE $\alpha$ membranaire et nucléaire}

La plupart des effets des œstrogènes sont relayés par le récepteur $\mathrm{R} \varepsilon \alpha$, un récepteur de la superfamille des récepteurs nucléaires qui exerce ses fonctions biologiques de plusieurs façons [1]. Classiquement, les œstrogènes se lient à leurs récepteurs, entraînant des changements conformationnels qui sont suivis par leur dimérisation et le recrutement de coactivateurs dans le noyau de la cellule où ils interagissent soit directement avec des éléments de réponse aux œstrogènes présents sur l'ADN, soit indirectement via la liaison à d'autres facteurs de transcription qui régulent la transcription de gènes cibles. Cette activité transcriptionnelle du $R \varepsilon \alpha$ repose sur l'action de deux fonctions d'activation (AF) distinctes, AFl et AF2.

Des modèles de souris ont été développés pour évaluer la contribution relative de chaque récepteur: $R \varepsilon \alpha$

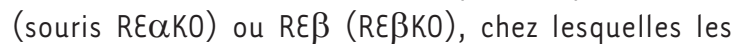
fonctions d'activation AFl $\left(R E \alpha-A F l^{0}\right)$ et AF2 (RE $\alpha$ $A F 2^{\circ}$ ) ont été inactivées [1]. L'utilisation des souris REßKO a montré le rôle mineur du récepteur RE $\beta$ dans la plupart des effets induits par les œstrogènes, ceuxci semblant surtout importants au niveau du système nerveux central et de la fertilité. En revanche, les études des souris déficientes en RE$\alpha A F l$ ont permis de démon-

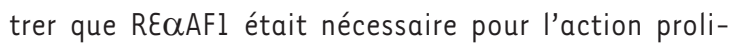
férative du $17 \beta$ estradiol (દ2) sur l'utérus et la glande mammaire. En revanche, RE$\alpha A F l$ n'est pas nécessaire pour observer les effets protecteurs sur les vaisseaux (athéroprotection), le diabète de type 2 et la déminéralisation de l'os (surtout cortical) [32]. Les souris $R \varepsilon \alpha-A F 2^{0}$, exprimant un RE $\alpha$ dépourvu d'AF2, perdent en fait toute action nucléaire de $R \varepsilon \alpha$, en raison de la dépendance de la fonction AFl vis-à-vis d'AF2 [33, 34] (Figure 1). Ces modèles animaux qui ont été développés sont également de précieux outils pour comprendre les mécanismes d'action des SERM. Les souris RE $\alpha-A F{ }^{0}$ ont ainsi permis de démontrer in vivo le rôle crucial de la fonction AFl dans les actions agonistes du tamoxifène sur l'utérus, sur la prévention de l'athérome et sur certains désordres métaboliques $[35,36]$.

Outre leur action génomique nucléaire classique, les œstrogènes induisent des effets rapides, dans les minutes qui suivent leur administration. Ces effets sont relayés par une sous-population de récepteurs qui sont exprimés à la membrane plasmique, un processus habi- 

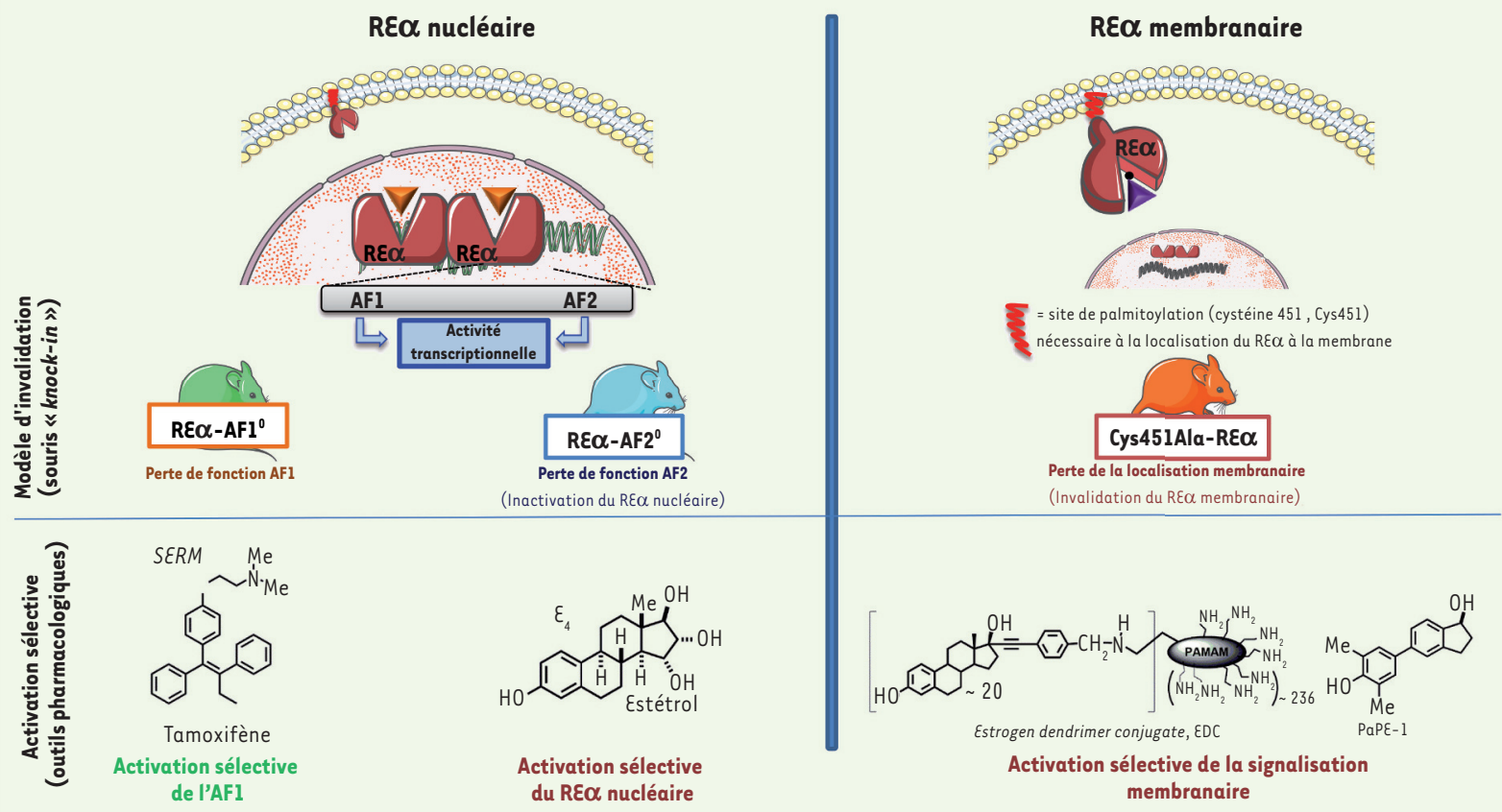

Figure 1. Modèles murins d'invalidation du récepteur des œstrogènes membranaire ou nucléaire et outils pharmacologiques d'activation sélective du $R \varepsilon \alpha$.

tuellement appelé « signalisation stéroïdienne initiée à la membrane » (MISS), ou «non génomique » ou encore « extranucléaire ». Pour évaluer directement in vivo le rôle respectif de l'action membranaire de $R \varepsilon \alpha$, par rapport à son action nucléaire, nous avons récemment généré un modèle de souris knock-in en remplaçant la cystéine en position 451 du $R \varepsilon \alpha$, site de sa palmitoylation, par une alanine (les souris sont désignées (451A-દR $\alpha$ ) [34] (Figure 1). II devient donc possible de distinguer l'activité des SERM sur le RE $\alpha$ nucléaire et sur le RE $\alpha$ membranaire.

II conviendrait dans un premier temps de définir systématiquement l'impact des SERM classiques (tels que le tamoxifène ou le raloxifène) sur les RE membranaires et nucléaires car leurs actions ont été décrites jusqu'alors comme une interférence avec les coactivateurs et les corépresseurs exprimés spécifiquement ou préférentiellement dans chaque tissu, et donc détaillés en ce qui concerne leurs actions nucléaires.

Des molécules sont d'ores et déjà disponibles permettant d'activer sélectivement le $R \varepsilon \alpha$ membranaire mais pas le $R \varepsilon \alpha$ nucléaire, ou inversement. J. Katzenellenbogen et ses collaborateurs ont mis au point des outils pharmacologiques afin d'activer spécifiquement le RE $\alpha$ membranaire. L'EDC (dendrimère conjugué à un œstrogène) est constitué d'éthinyl-œstradiol lié à une macromolécule de dendrimère de polyamidoamine (PAMAM) via des liaisons résistantes à I'hydrolyse [37]. Par sa grande taille et sa charge, l'EDC ne peut entrer dans le noyau. Il est donc spécifique de la stimulation des réponses non génomiques extra-nucléaires [37]. L'administration in vivo d'EDC entraîne l'augmentation de la production d'oxyde nitrique et l'accélération de la réendothélialisation après une lésion artérielle, mais il n'a pas d'impact sur la croissance utérine ou sur la prolifération de cellules de cancer du sein implantées chez la souris immunodéficiente [38]. Ce SERM est donc un outil pharmacologique qui pourrait fournir une protection vasculaire, sans augmenter le risque de cancer de l'utérus ou du sein, en stimulant sélectivement le $R \varepsilon \alpha$ membranaire [1]. Sa synthèse et sa structure ne sont cependant pas adaptées à un usage clinique. De nouvelles petites molécules dérivées de la structure de l'œstradiol, appelées Pathway Preferential Estrogen (PaPE), ont été conçues. Elles partagent avec I'EDC le même profil d'action, c'est à dire une activation sélective de la signalisation RE $\alpha$ membranaire mais pas de la fonction nucléaire [39]. Les PaPE pourraient former des complexes très labiles avec le récepteur, suffisant pour activer sélectivement des kinases, par activation membranaire, mais trop transitoire pour soutenir des effets nucléaires [39]. Dans les tissus murins non reproducteurs, les PaPE entraînent certaines activités protectrices vasculaires ainsi que des effets métaboliques bénéfiques dans les tissus hépatique et adipeux [39]. Ces nouvelles molécules pourraient être proposées après la ménopause afin d'induire des effets favorables aux niveaux métabolique et vasculaire, avec un risque minoré pour les tissus reproducteurs. Cependant, leur efficacité pour soulager les symptômes climatériques reste inconnue (Figure 1). 
Des études pharmacologiques et cliniques récentes suggèrent que l'estétrol (દ4) devrait être considéré comme un candidat intéressant pour le THM [40]. Les travaux de notre équipe ont montré que l'£4 est

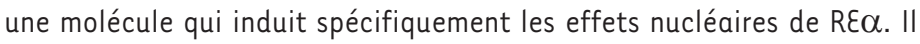
stimule l'expression des gènes de l'utérus, la prolifération épithéliale, et prévient l'athérome chez la souris, trois actions relayées par le RE $\alpha$ nucléaire, ce qui confirme les observations obtenues avec les modèles de souris transgéniques. L'E4 ne stimule cependant pas la NO synthase endothéliale, ni l'accélération de la cicatrisation endothéliale, deux processus qui dépendent uniquement de la signalisation induite par le RE $\alpha$ à la membrane [1]. L'E4 est naturellement produit à partir de l'દ2 et de l'œstriol (દ3) via la $15 \alpha$ - et la $16 \alpha$-hydroxylase par le foie fœtal humain pendant la grossesse. II rejoint la circulation maternelle via le placenta.

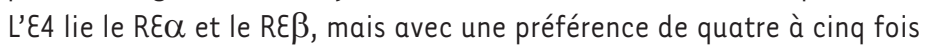
supérieure pour le $R \varepsilon \alpha$, avec cependant une affinité moindre que celle de l'éthinylestradiol (દદ) ou de l'દ2 [41]. Des expériences in vitro et in vivo ont montré que l'£4 nécessitait une dose 100 fois plus élevée que l'દ2 pour activer de manière similaire la transcription des gènes cibles par un mécanisme impliquant les fonctions d'activation d'AFl mais aussi celles d'AF2 [42]. Des études précliniques suggèrent que l'E4 s'oppose partiellement aux effets prolifératifs de l'£2 sur des cellules cancéreuses mammaires [43]. Il a également des activités œstrogéniques puissantes dans le cerveau, le tissu osseux, l'utérus et le vagin, et sur l'ovulation.

L'\&4 a une biodisponibilité orale élevée et une demi-vie plasmatique longue (environ $28 \mathrm{~h}$ chez la femme), ce qui autorise une administration orale une seule fois par jour [41]. Il a été évalué dans des études cliniques de phase I-II et s'est avéré avoir une bonne efficacité en contraception orale lorsqu'il était combiné avec un progestatif [44]. II est intéressant de noter que le traitement par l'£4 n'augmente pas les taux de facteurs de la coagulation et donc pourrait ne pas augmenter le risque de MTEV [40]. Il est actif pour prévenir les bouffées vasomotrices dans un modèle expérimental chez le rat [45], et une étude est actuellement en cours chez des femmes ménopausées (phase II) pour étudier son efficacité pour prévenir les bouffées de chaleur.

\section{Conclusion}

Les THM regroupent une large gamme de composés qui, chacun, montre des effets bénéfiques et des effets indésirables. L'indication du THM est actuellement principalement motivée par les symptômes climatériques impactant la qualité de vie et/ou la prévention de l'ostéoporose post-ménopausique chez la femme ayant un risque accru de fractures ostéoporotiques et présentant une intolérance ou une contreindication aux autres traitements indiqués dans la prévention de l'ostéoporose. Un THM est prescrit à la suite d'une évaluation individualisée du rapport bénéfice/risque selon les symptômes et les antécédents personnels et familiaux de la patiente. La molécule choisie et la voie d'administration doivent être en accord avec les objectifs du traitement, les préférences de la patiente et ses facteurs de risque.

Le développement clinique de nouveaux outils pharmacologiques sélectifs qui activent spécifiquement le RE $\alpha$ nucléaire ou membranaire est une étape importante. Il est probable que la spécificité tissulaire des SERM implique un effet sur le RE $\alpha$ membranaire et cette nouvelle dimension pourrait contribuer à expliquer la spécificité tissulaire et donc le rapport bénéfice/risque de ces molécules ou de ces associations de molécules.

La compréhension des différents mécanismes d'action des œstrogènes et des SERM est un enjeu médical important et pourrait aider à développer in fine un SERM « optimal » préservant les effets bénéfiques des œstrogènes sur les symptômes du climatère, le système urogénital, l'os et, si possible, prévenant le développement de l'athérome ou du diabète de type 2 et réduisant leurs effets indésirables, principalement un risque accru de thrombose veineuse, d'AVC, et de cancer du sein. $\diamond$

\section{SUMMARY}

Towards an optimization of the modulation of the estrogen receptor during menopausal hormonal therapy Women now live more than a third of their lives after the onset of menopause. The decline in endogenous estrogen production during this period is accompanied by functional disorders that affect quality of life. These symptoms may be relieved by menopausal hormone therapy (MHT) initially based on the administration of equine conjugated estrogens (mainly in the United States, oral route) or the natural estrogen, $17 \beta$-estradiol (in Europe, transdermal route). Estrogen receptor $\alpha(\varepsilon R \alpha)$, but not $\varepsilon R \beta$, mediates most of the physiological effects of estrogens. $\varepsilon$ R $\alpha$ belongs to the superfamily of nuclear receptors and regulates the transcription of genes via its activation functions AFl and AF2. In addition to these classical genomic actions, estrogens can activate a subpopulation of $\varepsilon R \alpha$ present at the cell membrane and thereby induce rapid signals. In this review, we will summarize the evolution of MHTs in last decades, as well as treatments that use various selective estrogen receptor modulators (SERMs). Next, we will describe recent advances in the understanding of the mechanisms of estrogen action, in particular the respective roles of nuclear and membrane $\varepsilon R \alpha$ as well as the potential implications for future therapies. $\diamond$

\section{LIENS D'INTÉRÊT}

Les auteurs déclarent n'avoir aucun lien d'intérêt concernant les données publiées dans cet article.

\section{RÉFÉRENCES}

1. Arnal JF, Lenfant F, Metivier R, et al. Membrane and nuclear estrogen receptor alpha actions: From tissue specificity to medical implications. Physiol Rev $2017 ; 97:$ 1045-87.

2. Stuenkel CA, Davis SR, Gompel A, et al. Treatment of symptoms of the menopause: an endocrine society clinical practice guideline. J Clin Endocrinol Metab 2015 ; 100 : 3975-4011. 


\section{RÉFÉRENCES}

3. Portman DJ, Gass ML, Vulvovaginal atrophy terminology consensus conference panel. genitourinary syndrome of menopause: new terminology for vulvovaginal atrophy from the international society for the study of women's sexual health and the North American menopause society. Menopause $2014 ; 21: 1063-8$

4. de Villiers TJ, Pines A, Panay N, et al. Updated 2013 International Menopause Society recommendations on menopausal hormone therapy and preventive strategies for midlife health. Climacteric $2013 ; 16: 316-37$.

5. Behl C. Oestrogen as a neuroprotective hormone. Nat Rev Neurosci 2002 ; $3: 433-42$.

6. Rossouw JE, Anderson GL, Prentice RL, et al. Risks and benefits of estrogen plus progestin in healthy postmenopausal women: principal results from the Women's Health Initiative randomized controlled trial. JAMA $2002 ; 288: 321-33$.

7. Anderson GL, Limacher M, Assaf AR, et al. Effects of conjugated equine estrogen in postmenopausal women with hysterectomy: the Women's Health Initiative randomized controlled trial. JAMA $2004 ; 291$ : 1701-12.

8. Lobo RA. Hormone-replacement therapy: current thinking. Nat Rev Endocrinol $2017 ; 13: 220-31$.

9. Schierbeck LL, Rejnmark L, Tofteng CL, et al. Effect of hormone replacement therapy on cardiovascular events in recently postmenopausal women: randomised trial. BMJ 2012 ; 345 : e6409.

10. Scarabin Py. Hormones and venous thromboembolism among postmenopausal women. Climacteric $2014 ; 17$ Suppl $2: 34-7$.

11. Renoux C, Dell'aniello S, Garbe $\varepsilon$, Suissa S. Transdermal and oral hormone replacement therapy and the risk of stroke: a nested case-control study. BMJ $2010 ; 340$ : c2519.

12. Tchaikovski SN, Rosing J. Mechanisms of estrogen-induced venous thromboembolism. Thromb Res $2010 ; 126: 5-11$

13. Olie V, Plu-Bureau G, Conard J, et al. Hormone therapy and recurrence of venous thromboembolism among postmenopausal women. Menopause $2011 ; 18: 488-93$.

14. Komm BS, Mirkin S, Jenkins SN. Development of conjugated estrogens/bazedoxifene, the first tissue selective estrogen complex (TSEC) for management of menopausal hot flashes and postmenopausal bone loss. Steroids $2014 ; 90: 71-81$.

15. Cuzick J, DeCensi A, Arun B, et al. Preventive therapy for breast cancer: a consensus statement. Lancet Oncol $2011 ; 12: 496-503$.

16. Grainger DJ, Schofield PM. Tamoxifen for the prevention of myocardial infarction in humans: preclinical and early clinical evidence. Circulation $2005 ; 112$ : 3018-24.

17. D'Amelio P, Isaia GC. The use of raloxifene in osteoporosis treatment. Expert Opin Pharmacother $2013 ; 14: 949-56$

18. Martinkovich S, Shah D, Planey SL, Arnott JA. Selective estrogen receptor modulators: tissue specificity and clinical utility. Clin Interv Aging $2014 ; 9$ : 1437-52.

19. Vogel VG, Costantino JP, Wickerham DL, et al. Effects of tamoxifen vs raloxifene on the risk of developing invasive breast cancer and other disease outcomes: the NSABP Study of Tamoxifen and Raloxifene (STAR) P-2 trial. JAMA $2006 ; 295$ : 2727-41.

20. Kim JH, Meyers MS, Khuder SS, et al. Tissue-selective estrogen complexes with bazedoxifene prevent metabolic dysfunction in female mice. Mol Metab $2014 ; 3$ : 177-90.

21. Komm BS, Kharode YP, Bodine PV, et al. Bazedoxifene acetate: a selective estrogen receptor modulator with improved selectivity. Endocrinology 2005 ; 146 : 3999-4008.

22. Lewis-Wambi JS, Kim H, Curpan R, et al. The selective estrogen receptor modulator bazedoxifene inhibits hormone-independent breast cancer cell growth and down-regulates estrogen receptor alpha and cyclin D1. Mol Pharmacol 2011; $80: 610-20$.

23. Buscato $M$, Fontaine $C$, Fabre $A$, et al. The antagonist properties of Bazedoxifene after acute treatment are shifted to stimulatory action after chronic exposure in the liver but not in the uterus. Mol Cell Endocrinol $2018 ; 472: 87-96$.

24. Silverman SL, Christiansen C, Genant HK, et al. Efficacy of bazedoxifene in reducing new vertebra fracture risk in postmenopausal women with osteoporosis: results from a 3-year, randomized, placebo-, and active-controlled clinical trial. J Bone Miner Res 2008 ; 23 : 1923-34.

25. Mirkin S, Komm BS. Tissue-selective estrogen complexes for postmenopausal women. Maturitas $2013 ; 76: 213-20$.

26. Harvey JA, Pinkerton JV, Baracat $\varepsilon C$, et al. Breast density changes in a randomized controlled trial evaluating bazedoxifene/conjugated estrogens. Menopause 2013 ; 20 : 138-45.

27. De Villiers TJ, Chines AA, Palacios S, et al. Safety and tolerability of bazedoxifene in postmenopausal women with osteoporosis: results of a 5 -year, randomized, placebo-controlled phase 3 trial. Osteoporos Int $2011 ; 22: 567-76$.
28. Skouby SO, Pan K, Thompson JR, et al. Effects of conjugated estrogens/ bazedoxifene on lipid and coagulation variables: a randomized placeboand active-controlled trial. Menopause $2015 ; 22$ : 640-9.

29. Chang KC, Wang Y, Bodine PV, et al. Gene expression profiling studies of three SERMs and their conjugated estrogen combinations in human breast cancer cells: insights into the unique antagonistic effects of bazedoxifene on conjugated estrogens. J Steroid Biochem Mol Biol 2010 ; 118 : 117-24.

30. Peano BJ, Crabtree JS, Komm BS, et al. Effects of various selective estrogen receptor modulators with or without conjugated estrogens on mouse mammary gland. Endocrinology 2009 ; 150 : 1897-903.

31. Komm BS, Mirkin S. Evolution of the tissue selective estrogen complex (TSEC). J Cell Physiol 2013 ; 228 : 1423-7.

32. Borjesson $A \varepsilon$, Windahl SH, Lagerquist MK, et al. Roles of transactivating functions 1 and 2 of estrogen receptor-alpha in bone. Proc Natl Acad Sci USA $2011 ; 108: 6288-93$

33. Billon-Gales A, Krust A, Fontaine C, et al. Activation function 2 (AF2) of estrogen receptor-alpha is required for the atheroprotective action of estradiol but not to accelerate endothelial healing. Proc Natl Acad Sci USA $2011 ; 108$ : 13311-6.

34. Adlanmerini M, Solinhac R, Abot A, et al. Mutation of the palmitoylation site of estrogen receptor alpha in vivo reveals tissue-specific roles for membrane versus nuclear actions. Proc Natl Acad Sci USA 2014 ; 111 : ع283-90.

35. Guillaume M, Handgraaf S, Fabre A, et al. Selective activation of estrogen receptor alpha activation function-l is sufficient to prevent obesity, steatosis, and insulin resistance in mouse. Am J Pathol 2017 ; 187 : 1273-87.

36. Fontaine C, Abot A, Billon-Gales A, et al. Tamoxifen elicits atheroprotection through estrogen receptor alpha AF-1 but does not accelerate reendothelialization. Am J Pathol 2013 ; 183 : 304-12.

37. Harrington WR, Kim SH, Funk CC, et al. Estrogen dendrimer conjugates that preferentially activate extranuclear, nongenomic versus genomic pathways of estrogen action. Mol Endocrinol $2006 ; 20: 491-502$.

38. Chambliss KL, Wu $Q$, Oltmann $\mathrm{S}$, et al. Non-nuclear estrogen receptor alpha signaling promotes cardiovascular protection but not uterine or breast cancer growth in mice. J Clin Invest $2010 ; 120: 2319-30$.

39. Madak-Erdogan Z, Kim SH, Gong P, et al. Design of pathway preferential estrogens that provide beneficial metabolic and vascular effects without stimulating reproductive tissues. Sci signal $2016 ; 9$ : ra53.

40. Mawet M, Maillard C, Klipping C, et al. Unique effects on hepatic function, lipid metabolism, bone and growth endocrine parameters of estetrol in combined oral contraceptives. Eur J Contracept Reprod Health Care 2015 ; $20: 463-75$.

41. Coelingh Bennink HJ, Heegaard AM, Visser M, et al. Oral bioavailability and bone-sparing effects of estetrol in an osteoporosis model. Climacteric 2008 ; 11 Suppl $1: 2-14$.

42. Abot $A$, Fontaine $C$, Buscato $M$, et al. The uterine and vascular actions of estetrol delineate a distinctive profile of estrogen receptor alpha modulation, uncoupling nuclear and membrane activation. EMBO Mol Med $2014 ; 6$ : 1328-46.

43. Visser $M$, Kloosterboer HJ, Bennink HJ. Estetrol prevents and suppresses mammary tumors induced by DMBA in a rat model. Horm Mol Biol Clin Investig $2012 ; 9: 95-103$.

44. Apter D, Zimmerman $Y$, Beekman L, et al. Bleeding pattern and cycle control with estetrol-containing combined oral contraceptives: results from a phase II, randomised, dose-finding study (FIESTA). Contraception 2016 ; 94 : 366 73.

45. Holinka CF, Brincat M, Coelingh Bennink HJ. Preventive effect of oral estetrol in a menopausal hot flush model. Climacteric $2008 ; 11$ (suppl 1) : 15-21.

TIRÉS À PART

F. Lenfant

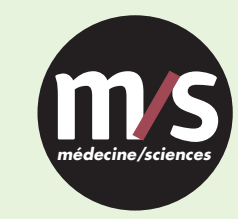

Tarifs d'abonnement $\mathrm{m} / \mathrm{s}-2018$

Abonnez-vous

à médecine/sciences
$>$ Grâce à $m / s$, vivez en direct les progrès des sciences biologiques et médicales

Bulletin d'abonnement page 1122 dans ce numéro de $\mathrm{m} / \mathrm{s}$ 